\title{
MARCAS REGISTRADAS, ESTRUCTURA DEL TEJIDO EMPRESARIAL Y COMPETITIVIDAD DEL NORTE DE SANTANDER ${ }^{1}$
}

\author{
TRADEMARKS, STRUCTURE OF THE BUSINESS TISSUE AND COMPETITIVENESS OF NORTE DE \\ SANTANDER
}

\author{
José Ascensión Cáceres Caballero ${ }^{2}$ \\ Universidad de Santander - Sede Cúcuta-Colombia
}

RECIBIDO: Noviembre 22 de 2015

ACEPTADO: Mayo 12 de 2016

\section{RESUMEN}

El presente es un artículo de reflexión cuyo objetivo consistió en analizar la información contenida en la investigación titulada "Estudio sobre el registro de marcas en productos y servicios y análisis del impacto en la ciudad de Cúcuta", relacionando los resultados con la situación que presenta el tejido empresarial y el índice de competitividad regional. El enfoque es cualitativo de tipo descriptivo porque se interpreta y reflexiona con base en información de fuentes documentales como el referido estudio sobre marcas, boletines del Observatorio Económico de la Cámara de Comercio, el Informe de la Superintendencia de Industria y Comercio y el Índice de Competitividad Departamental. La información de fuentes primarias fue recolectada, siguiendo técnicas de la metodología etnográfica, mediante entrevistas semi-estructuradas aplicadas a una muestra intencional de empresarios y gremios de los sectores estratégicos de la economía regional. Los resultados obtenidos señalan la correspondencia y reciprocidad existente entre las variables estudiadas, así como las principales debilidades observadas en la estructura del tejido empresarial, las dificultades en la evolución y dinámica empresarial y especialmente, el descenso en la posición competitiva del Norte de Santander durante los últimos años.

Palabras clave: competitividad, etnografía, informalidad, marcas, tecnología.

\begin{abstract}
This is a reflection article whose objective was to analyze the information compiled in the documentary source: "Study on registration and positioning of brands in products and services and impact analysis in the city of Cucuta" related to the current situation of the regional structure of the business field and competitiveness. The approach is qualitative-descriptive type, understood and reflected based on information from documentary sources such as the referred study on brands, the Annual Report of the Economic Observatory of the Chamber of Commerce of Cúcuta and the Regional Competitiveness Index. The information from primary sources was collected, following techniques of ethnographic methodology, through semi-structured interviews applied to an intentional sample of entrepreneurs and unions of the strategic sectors of the regional economy. The results obtained indicate the correspondence and reciprocity between the variables studied as well as the main weaknesses observed in the structure of the business network, the difficulties in the evolution and business dynamics and especially, the decrease in the competitive position of Norte de Santander during the last years.
\end{abstract}

Key words: Competitiveness, ethnography, informality, brands, technology.

\section{Este artículo se puede referenciar}

Cáceres, J. (2016). Marcas registradas, estructura del tejido empresarial y competitividad del norte de Santander. En Desarrollo Gerencial Revista de la Facultad de Ciencias Económicas Administrativas y Contables de la Universidad Simón BolívarColombia, 8(2), 76-93.

\footnotetext{
${ }^{1}$ Este artículo de reflexión se fundamentó en el desarrollo y resultados de la investigación titulada: "Estudio sobre el registro y posicionamiento de marcas en productos y servicios y análisis del impacto en la ciudad de Cúcuta”.
}

2 Magister Gerencia de Empresas, Docente tiempo completo, Universidad de Santander -Sede Cúcuta, cacerescaballero@gmail.com.

Desarrollo Gerencial, 8 (2) Pp. 76 - 93 Julio-Diciembre 2016. ISSN: 2145-5147 (On Line). Universidad Simón Bolívar. Barranquilla-Colombia. Contactos: desarrollogerencial@unisimonbolivar.edu.co 


\section{1.- INTRODUCCIÓN}

Dadas las condiciones de informalidad empresarial y laboral que caracteriza a la ciudad de Cúcuta y municipios del Área Metropolitana, La Universidad de Santander UDES de Cúcuta, consideró importante desarrollar una investigación para indagar sobre el estado de registro de marcas en productos y servicios como estrategia de la gestión industrial y comercial del empresariado de la región, relacionando este concepto con la estructura del tejido empresarial y el nivel de competitividad del departamento. El registro de marcas, el tejido empresarial y el índice de competitividad se constituyeron en las tres (3) variables fundamentales del estudio, sobre las cuales se logró consolidar información confiable y actualizada proveniente de la Superintendencia de Industria y Comercio, S.I.C. (2015), la Cámara de Comercio de Cúcuta y el Consejo Privado de Competitividad.

Los objetivos del referido estudio estuvieron centrados inicialmente en la realización de un proceso exploratorio con el fin de vencer las dificultades encontradas por la falta de información especialmente, con relación al registro de marcas. El alcance de la investigación fue cualitativo con características correlacionales, porque se analizó la relación entre las tres (3) variables y también tuvo alcance etnográfico de acuerdo con lo expuesto por Martínez (2000), ya que permitió obtener información confiable a través de las entrevistas realizadas a los empresarios y directivos de gremios de los distintos sectores estratégicos de la economía regional como son el sector de la arcilla, calzado y manufacturas de cuero, confecciones, comercio y servicios, alimentos, servicios tecnológicos y cosmetología.

Los entrevistados coincidieron en afirmar que una de las principales razones del bajo registro de marcas es la informalidad que caracteriza a la zona de frontera, donde el $96,1 \%$ de las unidades empresariales son microempresas y menos del $1 \%$ son empresas grandes y medianas; el $46 \%$ se dedican a la actividad comercial al por menor y mayor. Otro aspecto a considerar es que el $87 \%$ de las empresas afiliadas a la Cámara de Comercio están constituidas como persona natural y un 10\% como Sociedades de Acciones Simplificadas (S.A.S), según datos del Observatorio Económico de la Cámara de Comercio de Cúcuta (2015); situaciones que determinan la débil estructura del tejido empresarial de la ciudad.

Otros factores que explican el registro de marcas se relacionan con aspectos comportamentales característicos de los pequeños empresarios como el facilismo, la apatía y resistencia a trámites formales que les generen costos y los hace visibles en el medio. Igualmente señalan que a la S.I.C le falta visibilidad y promoción de los servicios. La evasión de impuestos y el creciente desarrollo de las marcas blancas por los comercializadores e intermediarios también fueron mencionados. Otro factor señalado es la falta de conocimientos para promover la innovación y modernización de las unidades empresariales.

Sobre el particular, Schütz (2011), presidente de Starbucks expresa sobre las razones de la marca que él estaba decidido a crear una empresa distinta, comprometida con hacer crecer el valor de sus 
acciones y con la responsabilidad fiscal de conseguir beneficios sin perder de vista el criterio de que para conseguir algo así hay que actuar a través de la conciencia social. Cerviño y Baena (2012), reconocen que, ante un entorno tan competitivo, muchas empresas han comprendido que la construcción de una marca fuerte, que condense una propuesta de valor para todos sus públicos, con seguridad tendrá ganado un camino muy corto para alcanzar una posición de privilegio, contara con un auténtico valor intangible y una fuerza importante en las decisiones de los compradores.

Los empresarios reconocen la contribución de una marca registrada en el posicionamiento de los productos y servicios, el fortalecimiento de la imagen corporativa y en la estabilidad económica de la empresa; opinaron que lo más importante es la protección y seguridad, el estatus, la confianza y la buena imagen.

El estudio presenta un análisis de la estructura del tejido empresarial que permite colegir sus efectos sobre el número de marcas registradas. De la misma forma se muestra un análisis del informe del Consejo Privado de Competitividad quien junto con el Centro de Pensamiento en Estrategias Competitivas, CEPEC (2015), de la Universidad del Rosario, elabora cada año el Índice Departamental de Competitividad -IDC-, en el cual se determina la posición del Norte de Santander a nivel nacional teniendo en cuenta entre varios factores, la innovación y la dinámica empresarial de cada región.

\section{2.- MÉTODO}

\section{$\checkmark$ Diseño}

El estudio tuvo una primera fase de investigación exploratoria consistente en la recopilación de documentos como bases de datos, informes institucionales, publicaciones a nivel de libros, artículos y proyectos de grado sobre las temáticas de marcas, estructura empresarial y competitividad. El diseño general fue de tipo descriptivo, con enfoque básicamente cualitativo con características correlacionales y etnográficas ya que se buscaba describir la forma como los empresarios desarrollan sus actividades gerenciales y conocer sus opiniones sobre las variables teniendo en cuenta los objetivos del estudio.

Para la recolección y análisis de información se utilizó las técnicas de la metodología etnográfica consistente en la realización de entrevistas semi-estructuradas al grupo de empresarios y gremios seleccionados. Para el análisis de los datos recolectados los distintos temas tratados en las entrevistas fueron clasificados en ocho (8) categorías con las siguientes denominaciones: \#1 Bajo registro de marcas; \#2 Ventajas y beneficios del registro de marcas; \#3 Innovación y desarrollo tecnológico, \#4 Capacidades del Recurso Humano; \#5 Apoyo gremial; \#6 Informalidad empresarial; \#7 Asociatividad Empresarial y \#8 Superación de la crisis. Estas categorías a su vez fueron agrupadas en subtemas con códigos, de acuerdo 
con las opiniones, ideas, comentarios o posiciones de los entrevistados; esta información dio lugar al planteamiento de las conclusiones del estudio.

\section{$\checkmark$ Participantes}

En primer lugar, se definió y segmentó la población objeto del estudio la cual quedó constituida por los empresarios y gremios de los sectores productivos registrados en la Cámara de Comercio de Cúcuta.

Se definió una muestra no probabilística o dirigida, de acuerdo con Hernández, Fernández y Baptista (2006), porque la población objeto del estudio fue clasificada por subsectores de acuerdo a características y perfil de las empresas según su tamaño, actividad económica y marcas registradas y considerando que para los propósitos de la investigación no se requería tanto la representatividad en cuanto a la cantidad de, unidades participantes en la muestra, sino la elección intencional de personas dentro de los segmentos definidos que se distinguieran por la trayectoria empresarial, conocimiento del tema y su disposición para participar en el entrega de información.

Se establecieron los siguientes criterios para la selección de las personas participantes de la muestra: pertenecer a una actividad industrial, comercial o de servicios, considerada estratégica dentro de la economía regional; ser empresa grande, mediana y pequeña; contar con marca registrada o no tener marca registrada en la S.I.C. Con relación a la escogencia de los representantes gremiales se aplicó el criterio de pertenecer a una actividad estratégica regional.

La muestra \#1 constituida por cuatro (4) empresarios con marcas registradas:

- Una (1) empresa de tamaño grande del sector Calzado y Manufacturas de cuero

- Una (1) empresa del tamaño grande del Sector de Alimentos productos lácteos

- Una (1) empresa de tamaño pequeño del sector de servicios de alimentos

- Una (1) empresa de tamaño pequeño de servicios agencia de viajes

Muestra \#2 constituida por cuatro (4) empresarios sin marcas registradas:

- Una (1) empresa de tamaño pequeño del sector de servicios de información tecnológica

- Una (1) empresa de tamaño mediano, del sector de arcilla y productos cerámicos

- Una (1) empresa de tamaño pequeño del sector de cosmetología

- Una empresa de tamaño pequeño del sector de confecciones

Muestra \#3 constituida por cuatro (4) directivos de gremios:

- INDUARCILLA

- ACICAM 
- Cámara de Comercio de Cúcuta

- FENALCO

\section{$\checkmark$ Instrumentos}

Se diseñaron y utilizaron cuatro (4) guiones de entrevistas, una para cada tipo de muestra, porque cada segmento de la población seleccionado tenía determinadas características que hacían necesaria esta distinción, por ejemplo, el tamaño de las empresas, las empresas con marcas registradas y las empresas sin marcas registradas, la entrevista a los gremios. Se logró comprobar que la utilización de la técnica de entrevista es el método más recomendado cuando se utiliza la metodología etnográfica.

Cada entrevista fue llevada a cabo personalmente por el investigador -joven investigador- y el docente tutor del proyecto, siendo grabada con registro fotográfico, previo consentimiento del entrevistado, y transcrita en forma fidedigna sin cambios en el contenido. Este material luego fue llevado al proceso de categorización y codificación de la información.

La validez y confiabilidad de la técnica de entrevista radicó fundamentalmente en la realización de una prueba piloto con un representante de cada grupo muestral que permitió hacer ajustes en el instrumento y el entrenamiento de los entrevistadores. El contexto de la entrevista, calidad de los empresarios y gremios participantes, el ambiente como se desarrollaron las conversaciones, la neutralidad del tema y espontaneidad de los participantes contribuyeron a la generación de un clima de confianza para la obtención de información objetiva y válida.

\section{$\checkmark$ Procedimientos}

Las etapas desarrolladas en todo el proceso de la investigación se pueden resumir de la siguiente forma:

1. Planeación de la investigación, trámites contractuales

2. Definición de la metodología, recolección y análisis de información secundaria, estudios, artículos, libros y compra de base de datos de la Cámara de Comercio de Cúcuta y de la S.I.C.

3. Definición de la Población, tamaño muestral, selección de participantes.

4. Diseño de la entrevista, guías de entrevista.

5. Organización del trabajo de campo.

6. Desarrollo de la prueba piloto

7. Etapa de promoción, contacto y concertación de las entrevistas con empresarios y gremios.

8. Ejecución del trabajo de campo- entrevistas-.

9. Transcripción y análisis de la información, categorización y codificación. 
10. Elaboración de informe técnico

11. Evento de divulgación y socialización de los resultados del estudio.

12. Elaboración de artículo.

\section{4.- RESULTADOS}

\section{$\checkmark \quad$ Análisis de marcas registradas en la S.I.C.}

En la tabla 1, se observa como varios departamentos con un número menor de marcas registradas ocuparon en el año 2015 una mejor posición que el Norte de Santander en el IDC, como el Quindío con 595 marcas (puesto 12) ocupó el puesto 11 y el puesto 9 en Innovación y Dinámica Empresarial; el Meta con 441 marcas (puesto 14) se ubicó en el puesto 10 y el puesto 11 en Innovación y Dinámica Empresarial; se destaca a Boyacá con el puesto 16 en número de marcas, el puesto 13 en Innovación y Dinámica Empresarial y el puesto 9 en el IDC . Ocho (8) departamentos que ocupan los primeros lugares en número de marcas también son los primeros en el índice.

Tabla 1. Comparativo de marcas registradas en la S.I.C. por departamentos y según el IDC.

\begin{tabular}{|c|c|c|c|c|}
\hline Departamentos & $\begin{array}{l}\text { Puesto } \\
\text { IDC }\end{array}$ & $\begin{array}{c}\text { Innovación y } \\
\text { dinámica empresarial } \\
\text { IDC }\end{array}$ & $\begin{array}{l}\text { Posición } \\
\text { Ocupada }\end{array}$ & $\begin{array}{l}\text { No. Marcas registradas } \\
\text { en la SIC }\end{array}$ \\
\hline Cundinamarca (incluye Bogotá) & 1 & 1 & 1 & $147.422(* *)$ \\
\hline Antioquia & 2 & 2 & 2 & 37.188 \\
\hline Valle del Cauca & 5 & 4 & 3 & 19.997 \\
\hline Atlántico & 8 & 8 & 4 & 5.516 \\
\hline Santander & 4 & 6 & 5 & 3.810 \\
\hline Risaralda & 6 & 7 & 6 & 2.209 \\
\hline Caldas & 3 & 3 & 7 & 1.774 \\
\hline Bolívar & 12 & 10 & 8 & 1.480 \\
\hline Cauca & 15 & 15 & 9 & 930 \\
\hline Tolima & 14 & 14 & 10 & 871 \\
\hline Norte de Santander & 17 & 17 & 11 & 830 \\
\hline Quindío & 11 & 9 & 12 & 595 \\
\hline Huila & 16 & 16 & 13 & 446 \\
\hline Meta & 10 & 11 & 14 & 441 \\
\hline Nariño & 13 & 20 & 15 & 402 \\
\hline Boyacá & 9 & 13 & 16 & 287 \\
\hline Magdalena & 21 & 12 & 17 & 273 \\
\hline Sucre & 18 & 21 & 18 & 247 \\
\hline Córdoba & 20 & 19 & 19 & 220 \\
\hline La guajira & 23 & 25 & 20 & 164 \\
\hline Cesar & 19 & 18 & 21 & 150 \\
\hline San Andrés y Prov. & -- & -- & 22 & 103 \\
\hline Casanare & -- & -- & 23 & 100 \\
\hline Caquetá & 22 & 22 & 24 & 48 \\
\hline Putumayo & 24 & 23 & 25 & 48 \\
\hline Choco & 25 & 24 & 26 & 28 \\
\hline Amazonas $(*)$ & -- & -- & 27 & 17 \\
\hline Arauca (*) & -- & -- & 28 & 11 \\
\hline Guainía (*) & -- & -- & 29 & 4 \\
\hline Vaupés (*) & -- & -- & 30 & 2 \\
\hline
\end{tabular}




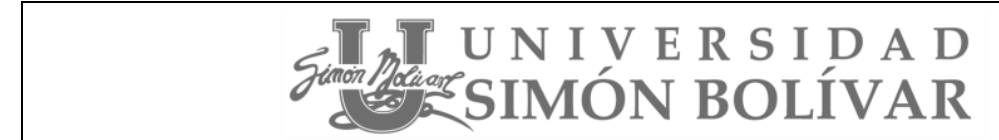

Guaviare (*)

Vichada (*)

Total

$\begin{array}{lll}-- & - \\ -- & -\end{array}$

Nota: (*) Departamentos que no reportaron datos en el IDC.

Fuente: Base de datos de la S.I.C. Datos ajustados por autor del artículo.

Norte de Santander ocupó el puesto 17 en el Índice Departamental de Competitividad IDC año 2015; el puesto 17 en el pilar de Innovación y Dinámica Empresarial y el puesto 11 con 830 marcas registradas sobre un total de 225.572 a nivel nacional, de las cuales 600 son de productos $(72,29 \%)$ y 230 son de servicios $(27,71 \%)$.

La actividad productiva que registra la mayor cantidad de marcas registradas es la de prendas de vestir y calzado con 221 marcas (26,63\%), seguida de la fabricación de productos químicos para distintos tipos de industrias con 82 marcas $(9,88 \%)$ y la de café, té, cacao, productos de panadería, lácteos, helados con 63 marcas $(7,59 \%)$.

En los servicios las empresas dedicadas a la publicidad, explotación, dirección y administración de negocios comerciales, ocupan el primer lugar con 80 marcas $(9,6 \%)$, le siguen los servicios de alimentación y hospedaje con 46 marcas (5,5\%); Educación, entretenimiento, actividades culturales y deportivas con 24 marcas (2,9\%); los servicios científicos y tecnológicos, de investigación con 18 marcas $(2,16 \%)$ y las actividades de seguros, monetarias y financieras con 15 marcas $(1,80 \%)$.

Estos resultados confirman la relevancia que tienen las actividades de producción de calzado y marroquinería, los servicios de alimentación y hospedaje, los servicios de comercio al por mayor y por menor, los servicios de seguros, monetarios y de banca, educación y entretenimiento, como las mas representativas en cuanto al número de marcas registradas pero al mismo tiempo, las de mayor crecimiento y desarrollo en el tejido empresarial de la región.

\section{$\checkmark \quad$ Estructura del tejido empresarial}

De acuerdo con el glosario del informe, Tejido Empresarial-Fortalecemos lazos en la región (2014), de la Cámara de Comercio de Cúcuta, los tejidos empresariales son el entramado de actores económicos, institucionales y culturales que dan origen a las actividades económicas de determinada región.

Para Crissien, (2008), de la escuela de Administración de Negocios EAN, "un tejido empresarial de calidad es aquel donde las empresas nacionales se fortalecen y pasan de ser buenas empresas, ya sean pequeñas o medianas, a ser empresas multinacionales aportando al Producto Interno Bruto (PIB), a la generación de empleo y competitividad internacional del país" (p25). 
Los datos suministrados por el Observatorio Económico de la Cámara de Comercio de Cúcuta (2015), señalan un crecimiento del 17\% en las empresas vigentes a diciembre de 2015 con relación al 2014, al pasar de 30.846 a 36.196 empresas registradas. La diferencia de 5.350 empresas nuevas registradas se explica en las empresas constituidas como persona natural y como unipersonales las cuales pasaron de 27.221 a 31.582 registros, o sea, 4.361 registros nuevos con un $16 \%$ de crecimiento.

La tabla 2 muestra la información por actividades que permite identificar los sectores con mayor peso en la estructura empresarial. La actividad económica de mayor peso corresponde a comercio al por mayor y al por menor, el 46\% de los negocios son de este sector (16.521 registros), seguido de la industria manufacturera con el 14\% (5.290 registros) y, en tercer lugar, otro sector de la economía terciaria, el alojamiento y servicios de comida aportando el $8 \%$ equivalente a 2.977 registros.

Las actividades de mayor nivel de crecimiento en cuanto a la creación y registro de nuevos negocios fueron la industria manufacturera con 3.052 unidades empresariales; la actividad profesional y científica con 426; la industria de la construcción con 384; el transporte y las comunicaciones con 342 y la actividad comercial con 231 negocios nuevos.

Las microempresas conforman el 96,1\% de la estructura empresarial de la ciudad y la participación de empresas grandes y medianas no alcanza el $1 \%$ del total de empresas.

Tabla 2 Clasificación de empresas por actividad económica al 31 de diciembre de 2014- 2015

\begin{tabular}{lccc}
\hline \multicolumn{1}{c}{ Actividad económica } & 31 dic & 31 dic & Variación \\
\hline Comercio & 2014 & 2015 & $1 \%$ \\
Industria manufacturera & 16.290 & 16.521 & $136 \%$ \\
Alojamiento y servicios. de & 2.238 & 5.290 & $-6 \%$ \\
Comida & 3.176 & 2.977 & $33 \%$ \\
Construcción & & & $38 \%$ \\
Activ. profesional y científica & 1.167 & 1.551 & $10 \%$ \\
Otras activ. de servicios & 1.119 & 1.545 & $-6 \%$ \\
Actividades de servicios & 1.318 & 1.448 & $50 \%$ \\
Transporte y comunicación & 1.202 & 1.128 & $38 \%$ \\
Información y comunicación & 682 & 1.024 & $16 \%$ \\
Actividades financieras & 579 & 797 & $30 \%$ \\
Otros & 661 & 769 & $17 \%$ \\
Total & 2.414 & 3.146 & \\
\hline
\end{tabular}

Fuente: Observatorio Económico Cámara de Comercio de Cúcuta (2015)

\section{$\checkmark \quad$ Índice Departamental de Competitividad año 2015}

Tradicionalmente los autores han definido la competitividad como la capacidad de un país para insertarse exitosamente dentro del mercado internacional pero mejorando al mismo tiempo el bienestar y calidad de vida de la población, tal como lo plantean Gutiérrez, López, Pérez y Rendón (2002), quienes 
entienden por competitividad la capacidad de un país para sostener y expandir su participación en los mercados internacionales y elevar simultáneamente el nivel de vida de la población, manifestando además que el desarrollo de las regiones requiere de algo más que de la voluntad del gobierno nacional, se necesita de los empresarios y los gremios.

Porter (1990), autor del libro La Ventaja Competitiva de las Naciones, dice que la productividad es el determinante más importante para la competitividad de una nación, destacando que las empresas de una nación deben mejorar inexorablemente la productividad de los sectores existentes mediante la calidad de los productos, la mejora de la propuesta de valor, la mejora de la tecnología del producto o superación de la eficiencia de la producción.

A su vez, Garay (1998), define la competitividad como la capacidad de los países para insertarse exitosamente en la economía mundial. Refiere que la competitividad de una nación es el grado al cual se puede producir bajo condiciones de libre mercado, bienes y servicios que satisfacen el test de los mercados internacionales y simultáneamente incrementar los ingresos reales de los ciudadanos. Jiménez (2006), considera que el principal problema que enfrentan las organizaciones actualmente, es la pérdida de mercados ante empresas cada vez más globalizadas, más eficientes y, por tanto, más competitivas.

El IMD (Institute Management Development), considera que la competitividad es el conjunto de indicadores políticas y factores que determinan la habilidad de crear y mantener un entorno en el cual las empresas continuamente aumenten el valor agregado de sus bienes y servicios y la población sea cada vez más prospera. En el Informe Nacional de Competitividad 2008, el Foro Económico Mundial -FEM- que se ha convertido en el principal referente en materia de competitividad a nivel mundial, afirma que la competitividad es el conjunto de instituciones políticas y factores que determinan la productividad de un país.

Saavedra (2012), hace referencia al Mapa de competitividad del BID y al modelo de competitividad sistémica del I.A.D. analizando los cuatro niveles planteados, pero mirándolos desde la óptica de país, región, industria y empresas, señalando su preocupación por que los autores según su parecer, se han preocupado por la competitividad empresarial dejando de lado el entorno que rodea a las empresas el cual directa o indirectamente influye en ella. De la misma forma, García, (2009) de la Universidad de Sonora, México, trata el concepto de la Competitividad Sistémica, señalando que los casos exitosos son explicados a partir de variables que muestran con claridad que el funcionamiento global del sistema es el que permite lograr una base sólida para el desarrollo de la competitividad; reconoce que el sistema además de requerir de esfuerzos individuales deben ser acompañados de innumerables aspectos que conforman el entorno de las organizaciones empresariales. 
El tema de la competitividad se aborda desde una perspectiva territorial teniendo en cuenta la incidencia que tiene el entorno geográfico sobre la gestión empresarial, la actividad económica y el bienestar de la población. Es así como el Índice de Competitividad Global, WEF (2015), por sus siglas en inglés, analiza la competitividad de 133 naciones, basándose en más de 110 indicadores que incluyen cifras oficiales provenientes de fuentes nacionales e internacionales y encuestas de opinión con líderes empresariales de todos los países del mundo; este índice explica las razones del por qué algunos países son más exitosos que otros, la productividad, los niveles de ingreso y las oportunidades para sus poblaciones.

Para los fines del presente análisis se extraen del IDC año 2015 los factores y pilares que tienen relación con el desarrollo empresarial y la gestión económica del departamento.

Tabla 3. Resumen de factores y posición IDC comparativa

\begin{tabular}{|l|c|c|c|}
\hline \multirow{2}{*}{ Posición comparativa IDC } & \multicolumn{3}{|c|}{ Puestos ocupados cada año } \\
\cline { 2 - 4 } & 2013 & 2014 & 2015 \\
\cline { 2 - 4 }
\end{tabular}

1. Condiciones básicas

\section{Factores}

2. Eficiencia

3. Sofisticación e innovación

$\begin{array}{lll}16 & 13 & 13 \\ 18 & 17 & 17 \\ 12 & 13 & 11\end{array}$

\section{Pilares}

Tamaño del mercado

$17 \quad 16$

Tamaño del mercado interno

$14 \quad 13 \quad 13$

Tamaño del mercado externo

Eficiencia de los mercados

Eficiencia del mercado de bienes

Eficiencia del mercado laboral

Desarrollo del mercado financiero

Sofisticación e innovación

Sofisticación

Diversificación

Innovación y dinámica empresarial

Investigación

Inversión en Ciencia, tecnología, innovación.

Dinámica empresarial

$\begin{array}{lll}17 & 14 & 13\end{array}$

Fuente: Datos del IDC 2015 - CEPEC. Resumido por autor del artículo.

Como dato neurálgico de los resultados del IDC se señala como el Norte de Santander en el año 2013 obtuvo la posición 14 entre todos los departamentos del país, habiendo perdido una posición en el año 2014 al pasar al puesto 15, pero en el año 2015 perdió dos (2) posiciones más al pasar al puesto 17. Dar una explicación precisa sobre las causas de esta caída es un asunto que requiere de un trabajo delicado y serio que no puede ser ligero por la cantidad de factores y variables que intervienen en la construcción del indicador. 
En los resultados encontrados se observan debilidades de manera especial en los pilares de Tamaño del Mercado que se calcula con base en el Producto Interno Bruto -P.I.B.-, las exportaciones y las importaciones; en el pilar Eficiencia de los Mercados que se refiere al mercado de bienes, mercado laboral y mercado financiero; igualmente se tienen dificultades en los temas de facilidades para abrir empresas, carga tributaria, la formalidad laboral, el desempleo, brechas de participación laboral de hombres y mujeres, la bancarización, entre varios componentes. De la misma manera en el pilar de Innovación y Dinámica Empresarial que comprende los aspectos de sofisticación del aparato productivo, la diversificación de los destinos de las exportaciones y de la canasta exportadora, además de las mediciones relacionadas con la tasa de natalidad y densidad empresarial y la participación de medianas y grandes empresas, los resultados logrados no son los mejores porque se ha perdido posicionamiento, con debilidades en las capacidades de los sectores productivos, empresariales e institucionales del Norte de Santander.

\section{5.- DISCUSIÓN}

Los empresarios y directivos de gremios reconocen la importancia, las ventajas y beneficios que tiene el registro de la marca ante la S.I.C., pero esta apreciación no se ve reflejada en la conformación de un tejido empresarial fuerte y competitivo como resultado de la influencia del número de marcas registradas en la Cámara de Comercio. Una de las razones que explican tal situación es la informalidad empresarial que se da en una zona de frontera sometida a vaivenes económicos, sociales y políticos, dando lugar a una alta permisividad consentida por la institucionalidad y sociedad en general.

La problemática detectada no es nueva, se reiteran las dificultades derivadas no solamente de la ubicación fronteriza que da lugar al surgimiento de operaciones comerciales y de todo tipo de negocios creados dentro del ambiente de la informalidad empresarial, sino también a problemas de orden social y cultural provenientes de la migración de personas hacia la frontera en búsqueda de trabajo, medios de subsistencia y en muchos casos de oportunidades para invertir capitales en negocios rentables llevando a la proliferación de establecimientos sin el lleno de los requisitos legales. Preocupante es la creencia que se volvió costumbre de que la frontera es un puerto libre para todo tipo de actividades, a costa de generar problemas de informalidad, inseguridad, pérdida de autoridad institucional, carencia de servicios básicos y el surgimiento de una fuerte competencia desleal y se puede decir que ilegal para la actividad formal generadora de riqueza.

En este sentido, Crissien (2008), afirma que un tejido empresarial... "débil o de poca calidad es el conformado por pequeñas empresas que no evolucionan y no crecen, que ante cualquier turbulencia internacional el país se convulsiona y estas empresas son muy vulnerables, no solo por la deficiencia en gerencia profesional sino también por la falta de competitividad" (p.25). Perea (2014), de la Universidad 
Jorge Tadeo Lozano, en el prólogo del resumen ejecutivo del estudio de Caracterización del Tejido Empresarial de Cúcuta hizo referencia a la importancia que tiene el tejido empresarial en el sentido de que este va más allá de las relaciones de encadenamiento productivo de la región o del proceso de aglomeración empresarial. Menciona que un tejido empresarial está compuesto por la relación que existe entre las empresas, las instituciones de orden local y de nivel regional, el territorio y la infraestructura con el tejido social y que, a partir de esta relación, es posible entender que las fuentes de competitividad y las limitaciones económicas de un municipio están estrechamente ligadas con los tipos de sociedades que lo habitan.

En otras palabras, se trata de un encadenamiento sostenido de sinergias donde juega papel principal la acción del gobierno, las capacidades del empresariado, la gestión de los gremios, la estructura y dinámica de los mercados, la participación efectiva de la academia y la sociedad. Cabra, G. (2013), señala el papel especial que tiene la academia dentro de este encadenamiento: "en la academia está el reto de la formación de emprendedores capaces de iniciar proyectos, de asumir riesgos y de propiciar el cambio y el crecimiento sostenible de la sociedad" (p.224).

El estudio demostró las debilidades que tiene el departamento con relación al número de marcas registradas en la S.I.C. dentro del ranking nacional, la estructura del tejido empresarial menguada por el alto porcentaje de micros y pequeñas empresas dedicadas a la actividad comercial, por su condición fronteriza y la preocupante situación resultado del descenso en dos (2) posiciones en el Índice Departamental de Competitividad año 2015, al pasar del puesto 15 al puesto 17. Es decir, se presentan dificultades a nivel de marcas, tejido empresarial y competitividad, las tres (3) variables del estudio. Se colige entonces una relación entre el número de marcas registradas, el estado del tejido empresarial y el índice de competitividad. Pero, se debe tener presente que el tejido empresarial está influenciado por otros factores que tienen que ver con el desarrollo empresarial y económico regional y el índice de competitividad se construye basado en una gran cantidad de factores que miden el avance del departamento en actividades, por ejemplo, salud, educación, infraestructura, ciencia y tecnología, tamaño del mercado, educación superior, desempeño administrativo y medio ambiente, entre otras actividades.

Sobre el concepto de competitividad, Peñaloza (2005), planteó que la competitividad empresarial y nacional es el resultado de la existencia de un conjunto de condiciones prevalecientes a lo largo de la historia económica y la misma está determinada por el modelo económico dominante, las capacidades de la empresa, la estructura de los mercados y la influencia del entorno, afirma que es el resultado de una combinación de factores y no de un factor único, por lo cual se puede aseverar que no existe una estrategia competitiva universal para tener éxito en los mercados, más bien depende de las características 
distintivas que ostente la organización o el país, en comunión con la comunidad del área de influencia, la tecnología y la productividad.

Por su parte, las marcas registradas son una señal de formalidad empresarial, de proyección de los negocios y capacidades gerenciales, el tejido empresarial resulta de la integración de actores institucionales de orden público y privado que representan y generan la actividad económica de la región. Trejos y Sanabria (2011), dicen que: "la marca constituye y constituirá una punta de lanza para el despegue económico de los comerciantes y sociedades mercantiles y que en el futuro las compañías que se concentren en hacer que un cliente piense primero en su marca, una vez que ha tomado la decisión de hacer una compra en esa categoría, serán las más exitosas" (p.113).

El autor resalta la importancia que tienen las relaciones que surgen de la gestión empresarial, la dinámica de las instituciones públicas y privadas y capacidades de la misma comunidad dentro de un territorio determinado. Reconoce que la competitividad y dificultades económicas de una región dependen de la comunidad y de las organizaciones públicas y privadas que la conforman. Torres (2015), considera que el tema del tejido empresarial es una tarea del sector público y del sector privado, que al estado le corresponde tomar la iniciativa en cuanto a la ciencia, la tecnología y la innovación y al sector privado construir y elaborar las relaciones empresariales, industriales, de nuevos emprendedores e innovadores y de inversionistas locales y extranjeros, dentro de un ambiente propicio con proyección hacia el futuro.

Por lo general todos los autores señalan la importancia y relación existente entre los referidos conceptos En resumen las marcas registradas significan visibilidad, proyección y transparencia empresarial, el tejido empresarial representa la unión e integración de fuerzas con objetivos comunes y la competitividad visibiliza brechas existentes, brinda resultados y señala puntos de partida para el mejoramiento de la región. Por lo tanto, es indiscutible la correspondencia que existe en estos conceptos. Es importante señalar entonces algunas ideas conducentes a mejorar la situación que se presenta a nivel del empresario, la empresa y la competitividad.

Montoya (2004), en su publicación se refiere al siguiente pensamiento de Joseph Schumpeter sobre la innovación: "El empresario es aquella persona que tiene capacidad e iniciativa para proponer y realizar nuevas combinaciones de medios de producción; es decir la persona que sea capaz de generar y gestionar innovaciones radicales dentro de las organizaciones o fuera de ellas" (p.211). Es importante insistir en estrategias de formalización empresarial para vencer el conformismo, la apatía y ponerle freno a la informalidad.

Los resultados demuestran que no es suficiente contar con una adecuada estructura desde el punto de vista del tamaño de las empresas, se requieren que sean competitivas, con capacidades gerenciales para la administración de los negocios, es necesario fortalecer las capacidades de las empresas con el fin de 
brindar una propuesta de valor innovadora, que favorezca la entrada a nuevos mercados y que ante las condiciones de vulnerabilidad de la zona de frontera garantice condiciones de sostenibilidad a mediano y largo plazo.

En cuanto a la competitividad se señalaron las variables que son tenidas en cuenta en la construcción del índice y que están directamente relacionadas con el desarrollo empresarial del departamento como el tamaño del mercado, la eficiencia de los mercados, la innovación y dinámica empresarial, las cuales demostraron debilidades que se reflejan en la débil estructura del tejido empresarial, más aún ahora por la actual situación de frontera.

Sin duda el sector económico más representativo de Cúcuta es el comercio, pero en esta actividad la innovación tiene espacio mediante la introducción de nuevos modelos de negocio y nuevas estrategias de comercialización. Las actividades de manufactura y servicios pueden optar por incorporar cambios en la propuesta de valor.

Con relación al diseño metodológico es necesario señalar que varios autores no están de acuerdo sobre la utilidad y beneficios del enfoque cualitativo y etnográfico y sobre el uso del muestreo no probabilístico de una investigación con determinación del tamaño de la muestra en forma dirigida o intencional, la experiencia y resultados de la presente investigación demuestran las bondades de este diseño metodológico de acuerdo con lo expresado por Hernández, Fernández C. y Baptista (2006), “ cuando se refieren al muestreo cualitativo en cuanto a que la decisión del tamaño y la selección de los miembros de la muestra es del investigador: "los estudios cualitativos son artesanales"... hechos a la medida de las circunstancias" (p.563).

Como se plantea más adelante, las instituciones públicas y privadas del departamento, han reaccionado a la problemática económica y social de la frontera con un plan de trabajo en desarrollo tendiente a la superación de la crisis, mediante acciones integrales que buscan crear condiciones estables para la región. Dada la importancia que tienen los conceptos de marcas, tejido empresarial y competitividad, se recomienda continuar realizando estudios sobre estas temáticas, monitoreando los indicadores y generando alertas sobre el comportamiento de los mismos, como insumo para los futuros planes de desarrollo a nivel institucional y gubernamental.

En este orden de ideas se presentan las siguientes conclusiones:

$\checkmark$ Se determinó que existe correspondencia y reciprocidad entre las variables estudiadas. Se observan fuertes debilidades en cada una de ellas, principalmente en actividades relacionadas con la gestión y dinámica empresarial. 
$\checkmark$ El registro de marcas que denota la capacidad del empresariado de trascender, proyectar y visibilizar una imagen para lograr posicionamiento en el mercado nacional y/o internacional, además de ser un efectivo aporte a la política regional de formalización empresarial, no muestra resultados halagadores.

$\checkmark \quad$ El tejido empresarial, no sólo analizado desde el punto de vista del número y tamaño de las empresas que lo conforman, sino especialmente, por su contribución al desarrollo y eficiencia de los mercados, la conformación de alianzas estratégicas y clústeres empresariales, la innovación y dinámica empresarial, presenta dificultades.

$\checkmark$ El Índice de Competitividad del Norte de Santander, muestra resultados preocupantes. El descenso del departamento durante los últimos años, del puesto 14 al puesto 17, dada la importancia y rigurosidad de los factores que intervienen y con los cuales se construye el indicador, merece un serio análisis, una profunda reflexión y una convocatoria general para reaccionar y evolucionar a todo nivel por parte de las instituciones públicas y privadas de diversa índole: gobierno, sectores productivos, gremiales, sociales, académicos, políticos, etc.|

Las causas esgrimidas y que en parte justifican la situación de rezago encontrada en el registro de marcas, la inexistencia de un sólido tejido empresarial y las brechas encontradas en el indicador de competitividad, hacen referencia a la informalidad empresarial, laboral y social de la frontera, que ha dado lugar a una alta concentración de microempresas, en gran proporción de la actividad comercial al por mayor y menor, en detrimento de la actividad manufacturera y del aprovechamiento de mercados alternativos a nivel nacional e internacional.

$\checkmark$ Las tres variables estudiadas son herramientas válidas para fijar metas en los planes de desarrollo del sector institucional, empresarial, gremial o sectorial, evaluar resultados y hacer nuevas proyecciones.

$\checkmark$ La utilización de técnicas de la metodología etnográfica contribuyó de manera significativa en el cumplimiento de los objetivos de la investigación al facilitar el tratamiento de la información por medio de entrevistas planeadas y concertadas con el grupo de empresarios y directivos de gremios representativos seleccionados por su idoneidad y disposición para suministrar los datos. Las características de los mismos, sus objetivos, los factores y cosas comunes que los unen y su localización dentro de una misma región permitió que fueran considerados y tratados como un grupo o comunidad social de índole empresarial.

$\checkmark$ La determinación de una muestra no probabilística, tomada en forma intencional o dirigida y definida con criterios previamente establecidos de acuerdo con los objetivos propuestos, de la misma forma, produjo favorables resultados para el logro de los objetivos de la investigación. 
$\checkmark$ Se señala como dificultad la presentada en la comparación de la base de datos de marcas registradas de la S.I.C y la base de datos de registros de la Cámara de Comercio, ya que la S.I.C. se rige por el Acuerdo de Niza, que establece una clasificación en productos y servicios y la Cámara de Comercio utiliza la clasificación Industrial Internacional Uniforme C.I.I.U, siendo dos sistemas muy diferentes para clasificar las actividades económicas, lo cual no permitió, un análisis más preciso de la información.

$\checkmark$ Como punto fuerte de la investigación se señala el conocimiento obtenido en las entrevistas de que los principales actores empresariales, institucionales y gremiales de la región son conscientes de la problemática y que ante los últimos acontecimientos de orden político, económico y social de Venezuela, están promoviendo un trabajo mancomunado para desarrollar tareas direccionadas a lograr propósitos comunes de acuerdo con los objetivos y características de cada organización, dentro de las cuales está el fortalecimiento de la alianza Universidad-Estado-Empresa.

\section{6.- REFERENCIAS}

Cabra, G. (2013). Micro, pequeña y mediana Empresa, la oportunidad de emprender. Revista Desarrollo Gerencial. Vol. 5 Núm. 1, p. 224. Universidad Simón Bolívar, Extensión Cúcuta: Colombia.

Cámara de Comercio de Cúcuta, Alianza Ecopetrol, Confecámaras, Universidad Jorge Tadeo Lozano y Corporación Calidad. (2014). Resumen ejecutivo de documento Tejido Empresarial-Fortalecemos lazos en la región. Editorial Difusión Compañía de Medios: Cúcuta.

CEPEC (2015). Índice Departamental de Competitividad, Edit. Puntoaparte. Bogotá, recuperado: www.compite.com.co/

Cerviño, J. \& Baena, V. (2012). Gestión Estratégica de la marca en el mundo del vino. Revista Spanish Journal Of Rural Development, Vol.3 Num. extra 17 (3), Madrid: España

Colciencias (2013). Convocatória \# 617, Programa Joven Investigador. Recuperado www.colciencias.gov.co.

Crissien, J. (2008). Círculo virtuoso de la educación empresarial: una propuesta de modelo para el desarrollo de un tejido empresarial de calidad. Revista Escuela de Administración de Negocios, EAN, 63, p.25 Bogotá: Colombia.

Foro Económico Mundial -FEM-, Índice de Competitividad Global año 2008, Davos: Suiza.

Garay, L. J. (1998), Colombia: Programa de Estudio. Estructura industrial e internacionalización 19671996, D.N.P -COLCIENCIAS- 1998, p. 560. Bogotá. 
García, G. (2009). El concepto de competitividad sistémica. Revista Universidad de Sonora, 29-31. México.

Gutiérrez, E., López, L., Pérez, J., y Rendón, A, (2002). Desarrollo regional: reflexiones y alternativas. Semestre Académico 5(9). P.3, Universidad de Medellín: Colombia.

Hernández, R., Fernández C. y Baptista, P. (2006). Metodología de la Investigación, p. 241 p. 563, 4 edición, Mc Graw Hill Interamericana. México. D.F.

Jiménez, M. H. (2006). Modelos de Competitividad empresarial. Umbral Científico, 9, p.p. 122.

Universidad Manuela Beltrán, Bogotá: Colombia.

La organización Mundial de la Propiedad Intelectual -OMPI- Administradora del Arreglo de Niza (1957). Recuperado: www.wipo.int/portal/es

Martínez, M. (2000). La investigación cualitativa etnográfica en investigación, manual teórico - práctico. P.38 Editorial Trillas, México.

Montoya, O. (2004). Schumpeter, innovación y determinismo tecnológico. Revista Scientia et Technica, 2 (25), p.211. Universidad Tecnológica de Pereira: Colombia.

Observatorio Económico de la Cámara de Comercio de Cúcuta (2015). Boletín Diciembre 2015, recuperado: $\underline{w w w . d a t a c u c u t a . c o m}$

Peñaloza, M. (2005). Competitividad: ¿nuevo paradigma económico? Revista Fórum Empresarial 10(1), p.50. Centro de Investigaciones comerciales e iniciativas académicas, San Juan de Puerto Rico.

Perea, G. (2014). Prólogo de la publicación de la Cámara de Comercio de Cúcuta, Caracterización del Tejido Empresarial de Cúcuta, p.5. Cúcuta

Porter, M. (1991). La ventaja Competitiva de las naciones. Barcelona: Plaza \& Janes Editores. p.28, Barcelona España.

Saavedra, M.L. (2012). Una propuesta para la determinación de la competitividad de la Pyme Latinoamericana. Revista Pensamiento y gestión, 33, 107-108, Universidad del Norte, Barranquilla: Colombia.

Schütz, H. (2011). Libro el Desafío de Starbucks. p.12, Editorial Santillana, $1^{\circ}$ Edición, Madrid: España. Superintendencia de Industria y Comercio. Base de Datos sobre marcas registradas en Colombia por ciudad de 1990 a 2013. 
Torres, L. (2015). Educación e innovación: pilares del desarrollo. Revista Propiedad Inmaterial, 20, p. 102, Universidad Externado de Colombia: Bogotá.

Trejos, K. \& Sanabria, C. (2011). El registro de marcas como plataforma para el crecimiento de una empresa. El Cotidiano.p.113, Universidad Autónoma Metropolitana de México D.F.

Universidad de Santander - UDES- de Cúcuta. Estudio sobre el registro y posicionamiento de marcas en productos y servicios y análisis del impacto en la ciudad de Cúcuta (2014-2015), Informe técnico de avance o final de Programas de Ciencia y Tecnología, presentado a COLCIENCIAS, Programa Joven

Cúcuta,

Colombia. 